\title{
Evaluation of missile electromagnetic launch system based on effectiveness
}

\author{
Qiaoyang Li*, Guiming Chen, Ziqi Li, Yi Zhang and Lingliang Xu \\ Xi'an Research Institute of High-Tech, Xi'an, China
}

\begin{abstract}
To solve the problems of strong infrared radiation, poor continuous combat capability of the system, serious ablation of the launching device, and environmental pollution of the existing missile launching system, electromagnetic launch system (EMLS) has been studied for missile launch system. Combining the situation that the current research on missile electromagnetic launch system (MEMLS) mainly focuses on the key technical points and the deficiencies in the previous research on MEMLS, this paper establishes an effectiveness prediction model based on GRA-PCA-LSSVM, and discusses the investment efficiency of the system based on DEA. The experimental results prove that the established model is reasonable, effective and superior, and provides a reference for the further improvement and development of MEMLS.
\end{abstract}

Keywords: MEMLS, Grey relation analysis (GRA), Principal component analysis (PCA), Least square support vector machine (LSSVM), Data Envelopment Analysis (DEA)

\section{Introduction}

Electromagnetic launch system (EMLS) is a launching technology that uses electromagnetic energy to convert it into payload kinetic energy [1]. EMLS can convert electrical energy into the kinetic energy required by the load in a short time, and push objects to reach a certain speed quickly [2]. Since it can effectively solve the problems of strong infrared radiation, poor continuous combat capability of the system, serious ablation of the launcher, and environmental pollution of the existing system, missile electromagnetic launch system (MEMLS) is a current research hotspot.

The current research on MEMLS concentrates on technical points such as pulse energy storage power supply, pulse power discharge, motor control, etc. There are few studies on the whole system evaluation. The author has studied the effectiveness evaluation method of MEMLS and proposed a new evaluation

\footnotetext{
*Corresponding author. Qiaoyang Li, Xi'an Research Institute of High-Tech, Xi'an, China. E-mail: liqiaoyang2019@163.com.
}

model for the two-level indicators of the system [3]. However, the system has many evaluation indicators and the model is quite complex, so the effectiveness value of the design scheme cannot be calculated quickly, and the investment efficiency of the design scheme is not discussed.

Aiming at the problem of too many evaluation indexes, Wang used gray correlation analysis (GRA) and support vector machine (SVM) to study and optimize the performance of asphalt pavement [4]; Yu proposed an improved principal component analysis (PCA) model to study the fault detection of nuclear power station sensors [5]. For the study of prediction model, Chung used multi-channel convolutional neural network optimized by genetic algorithm to predict the stock market [6]; Stoichev used multiple regression model to study the pollution of metals and quasi metals in surface sediments [7]; Liu used SVM model optimized by particle swarm optimization to analyze and predict PM2.5 [8]. To solve the problem of investment efficiency, Yeung used data envelopment analysis (DEA) to study the efficiency of Brazilian courts [9]; Yang used DEA to evaluate the 
efficiency of China's industrial waste gas treatment [10].

Existing research models only solve part of the problems, and there are few in the field of MEMLS. This paper evaluates MEMLS based on effectiveness, focusing on the rapid calculation of effectiveness model and system investment efficiency. The main innovations are as follows.

(1) From the perspective of the system, this paper conducts further research on the effectiveness calculation model of MEMLS, which makes up for the lack of most research focusing on specific technologies.

(2) This paper establishes the effectiveness calculation model of GRA-PCA-LSSVM, which can quickly calculate the effectiveness value of MEMLS.

(3) This paper uses the DEA model to study the investment efficiency of MEMLS, and proposes suggestions for improvement of the design scheme.

(4) This paper supplements the deficiencies of the previous research, which is innovative and practical.

The main structure of this paper is as follows: Section 1 introduces the basic concepts and advantages of EMLS and MEMLS, and points out the status and shortcomings of the current research of MEMLS; Section 2 establishes a model for fast calculation of effectiveness and investment efficiency of MEMLS; Section 3 combines the existing sample data to apply and verify the model, which proves the effectiveness of the model and methods; Section 4 summarizes this paper.

\section{System evaluation model based on effectiveness}

This section mainly introduces the model established in this paper for MEMLS evaluation. The steps and methods of model establishment are shown in Fig. 1.

The effectiveness evaluation model in Fig. 1 has been studied in the early stage [3], and is used directly as a model here.

\subsection{Effectiveness calculation model based on GRA-PCA-LSSVM}

To solve the problem that the original effectiveness evaluation model is too complicated, firstly establish a fast calculation model of effectiveness based on GRA-PCA-LSSVM, which is convenient to directly obtain the effectiveness value from the design scheme of MEMLS.

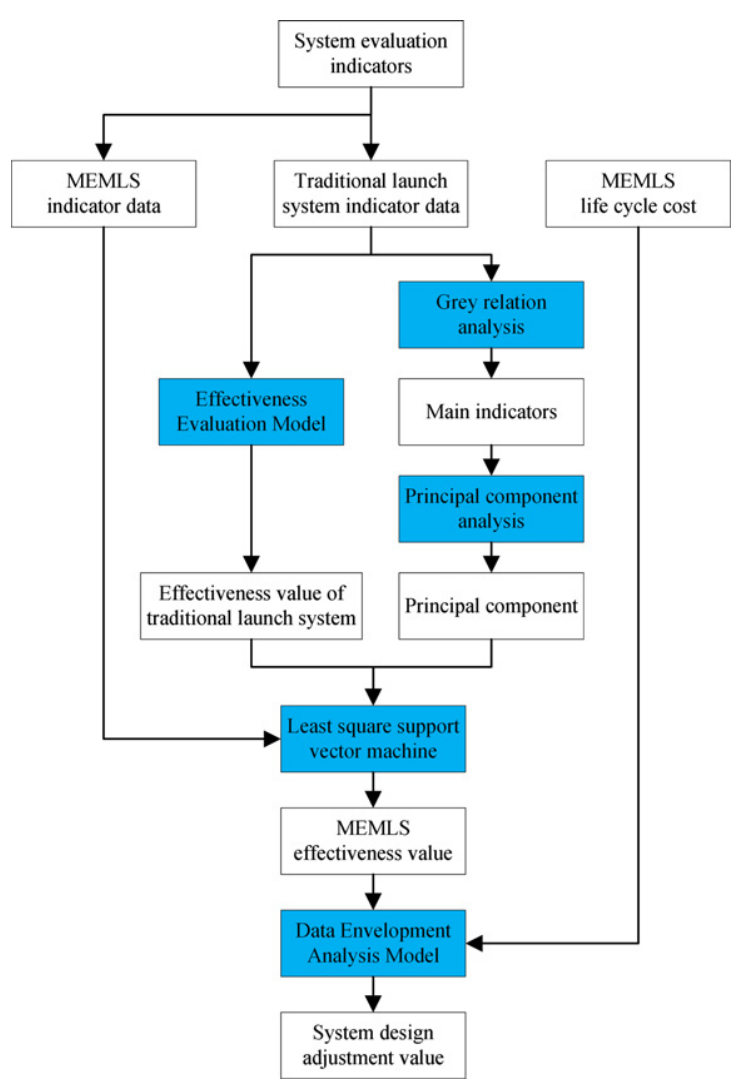

Fig. 1. Evaluation model based on effectiveness.

\subsubsection{GRA}

When evaluating the system, there are too many original evaluation indicators, which will lead to the redundancy of information and the complexity of the process. Therefore, it is necessary to select the main indicators. Grey system theory was founded in 1982 by Professor Deng Julong of China. It is a system control theory about the incomplete or uncertain internal system [11]. GRA is a multi-factor statistical analysis method in grey system theory, which judges the correlation degree according to the similarity degree of the changing trends between factors. Because of its simple calculation and low sample requirements, GRA has been widely used in industry, materials, and agriculture [12-14]. The steps of GRA are as follows.

Step 1: Determine the parent sequence and subsequence in the system. The parent sequence is a sequence that reflects the characteristics of the system's behavior, and the subsequence is a sequence that affects the characteristics of the system's behavior.

Step 2: Dimensionless processing of the parent sequence and subsequence. In the analysis process, 
different dimensions may lead to errors in the results. Generally, the sequence is processed by the averaging method to remove dimensions.

Step 3: Calculate the correlation coefficient of the factors corresponding to the sub-sequence and the parent sequence, as shown in formula (1).
Step 4: Select the principal component according to the specific requirements of the eigenvalue or the cumulative contribution rate of the principal component to complete the PCA. The general mathematical model of PCA results is shown in formula (3).

$$
\xi\left(x_{0}(k), x_{i}(k)\right)=\frac{\min _{i} \min _{k}\left|x_{0}(k)-x_{i}(k)\right|+\rho \max _{i} \max _{k}\left|x_{0}(k)-x_{i}(k)\right|}{\left|x_{0}(k)-x_{i}(k)\right|+\rho \max _{i} \max _{k}\left|x_{0}(k)-x_{i}(k)\right|},\left\{\begin{array}{l}
i=1,2, \cdots, m \\
k=1,2, \cdots, n
\end{array}\right.
$$

In the formula: $m$ is the number of subsequences; $n$ is the number of samples; $x_{0}(k)$ is the $k$-th sample value of the parent sequence after dimensionless processing; $x_{i}(k)$ is the $k$-th sample of the $i$-th subsequence after dimensionless processing Value; $\rho$ is the resolution coefficient, reflecting the size of the resolution, usually 0.5 .

Step 4: Calculate the degree of relevance and sort to obtain the degree of relevance of each sub-sequence to the parent sequence, as shown in formula (2).

$$
r_{i}=\frac{1}{n} \sum_{k=1}^{n} \xi\left(x_{0}(k), x_{i}(k)\right), i=1,2, \cdots, m
$$

\subsection{2. $P C A$}

PCA is a multivariate statistical analysis method that converts multiple interrelated indicators into a few comprehensive indicators. In the research of multiple indicators, Since there is often a certain correlation between the indicators, the data will overlap with information, which is more complicated for high-dimensional research. PCA adopts the method of dimensionality reduction, and uses a small number of comprehensive factors to express all the original indicators, and requires that the original indicator information is reflected as much as possible, and the factors are not related to each other. PCA has been widely used in environmental science, agriculture and industry [15-17]. The steps of PCA are as follows.

Step 1: Standardize the original data to eliminate the influence of different dimensions and orders of magnitude on the analysis results.

Step 2: Perform correlation analysis on the processed sample matrix to obtain the correlation coefficient matrix, and determine whether PCA can be performed.

Step 3: From the correlation coefficient matrix, the eigenvalue and variance are calculated by the Jacobian method, and the principal component variance contribution rate and cumulative contribution rate are calculated.

$$
\begin{aligned}
& Z_{1}=a_{11} X_{1}+a_{12} X_{2}+\cdots+a_{1 n} X_{n} \\
& Z_{2}=a_{21} X_{1}+a_{22} X_{2}+\cdots+a_{2 n} X_{n} \\
& \vdots \\
& Z_{l}=a_{l 1} X_{1}+a_{l 2} X_{2}+\cdots+a_{l n} X_{n}
\end{aligned}
$$

In the formula: $Z_{l}$ is the main component; $X_{n}$ is the normalized raw data; $a_{i j}$ is the main component coefficient.

\subsubsection{LSSVM}

Least Square Support Vector Machine (LSSVM) is an extension of SVM. LSSVM takes the quadratic loss function as the empirical risk, and replaces the inequality constraints with equality constraints, transforming the training of the model into the calculation of linear equations, reducing the computational complexity [18]. Because of its superiority, LSSVM is widely used in meteorology, materials science, industry and other fields [19-21]. The establishment process of the LSSVM model is as follows.

Suppose that the number of samples is $n, x_{i}$ is the $\mathrm{m}$-dimensional input vector, and $y_{i}$ is the output vector. Construct the optimal linear regression function as formula (4).

$$
f(x)=\omega^{\mathrm{T}} \varphi(x)+b
$$

In the formula: $\omega$ is the weight vector; $b$ is the offset; $\varphi(x)$ is the nonlinear mapping.

According to the principle of structural risk minimization, the objective function can be expressed as formula (5).

$$
\min \frac{1}{2} \omega^{\mathrm{T}} \omega+\frac{\lambda}{2} \sum_{i=i}^{n} e_{i}^{2}
$$

Where: $\lambda$ is the regularization parameter; $e_{i}$ is the prediction error vector of the training set. 
The constraint condition is formula (6).

$$
y_{i}=\omega^{\mathrm{T}} \varphi\left(x_{i}\right)+b+e_{i}
$$

The Lagrangian function is used to transform the problem into the dual space, as in formula (7).

$$
L=\frac{1}{2} \omega^{\mathrm{T}} \omega+\frac{\lambda}{2} \sum_{i=i}^{n} e_{i}^{2}-\sum_{i=1}^{n} \alpha_{i}\left[\omega^{\mathrm{T}} \varphi\left(x_{i}\right)+b+e_{i}-y_{i}\right]
$$

In the formula: $\alpha_{i}$ is the Lagrange multiplier. According to the KKT condition, the formula (8) can be obtained.

$$
\left\{\begin{array}{l}
\omega=\sum_{i=1}^{n} \alpha_{i} \varphi\left(x_{i}\right) \\
\sum_{i=1}^{n} \alpha_{i}=0 \\
\alpha_{i}=\lambda e_{i} \\
\omega^{\mathrm{T}} \varphi\left(x_{i}\right)+b+e_{i}-y_{i}
\end{array}\right.
$$
(9).

$$
\left[\begin{array}{cccc}
0 & 1 & \cdots & 1 \\
1 & K\left(x_{1}, x_{1}\right)+\frac{1}{c} & \cdots & K\left(x_{1}, x_{1}\right) \\
\vdots & \vdots & & \vdots \\
1 & K\left(x_{n}, x_{n}\right) & \cdots & K\left(x_{n}, x_{n}\right)+\frac{1}{c}
\end{array}\right]\left[\begin{array}{c}
b \\
\alpha_{1} \\
\vdots \\
\alpha_{n}
\end{array}\right]=\left[\begin{array}{c}
0 \\
y_{1} \\
\vdots \\
y_{n}
\end{array}\right]
$$

In the formula: $K\left(x_{i}, x_{j}\right)=<\varphi\left(x_{i}\right), \varphi\left(x_{j}\right)>$ is the kernel function. Generally take the radial basis kernel function as equation (10).

$$
K\left(x_{i}, x_{j}\right)=\exp \left(-\frac{\left\|x_{i}-x_{j}\right\|^{2}}{2 \mu^{2}}\right)
$$

In the formula: $\mu$ is the nuclear parameter.

Finally, the prediction model of LSSVM is

$$
y(x)=\sum_{i=1}^{n} \alpha_{i} K\left(x_{i}, x_{j}\right)+b
$$

\subsection{Evaluation of system investment efficiency based on DEA model}

The DEA method is a non-parametric method used to evaluate the relative effectiveness of decisionmaking units (DMUs) with the same type of multiple inputs and multiple outputs. At present,
DEA research is relatively mature and widely used in economics, sociology and industry [22-24].

\subsubsection{Overall effectiveness evaluation model (CCR)}

Suppose there are a total of $s$ DMUs, each DMU has $p$ inputs and $q$ outputs, $x_{k}=\left(x_{1 k}, x_{2 k}, \cdots, x_{p k}\right)^{\mathrm{T}}$ represents the input vector of the $k$-th DMU, $y_{k}=$ $\left(x_{1 k}, x_{2 k}, \cdots, x_{q k}\right)^{\mathrm{T}}$ represents the output vector of the $k$-th DMU, and $x_{0}$ and $y_{0}$ represent the input and output vectors of the $\mathrm{DMU}_{0}$. From the literature [25], the BCC model is:

$$
\left\{\begin{array}{c}
\min \theta \\
\text { s.t. } \sum_{k=1}^{s} \lambda_{k} x_{k}+s^{-}=\theta x_{0} \\
\sum_{k=1}^{s} \lambda_{k} x_{k}-s^{+}=y_{0} \\
s^{-} \geq 0, s^{+} \geq 0
\end{array}\right.
$$

In the formula: $s^{-}$and $s^{+}$are slack variables; $\lambda_{k}$ is a general variable.

To simplify the calculation, the non-Archimedean infinitesimal quantity is introduced, then the formula (12) becomes

$$
\left\{\begin{array}{c}
\min \left[\theta-\varepsilon\left(\hat{e}^{\mathrm{T}} s^{-}+\hat{e}^{\mathrm{T}} s^{+}\right)\right] \\
\text {s.t. } \sum_{k=1}^{s} \lambda_{k} x_{k}+s^{-}=\theta x_{0} \\
\sum_{k=1}^{s} \lambda_{k} x_{k}-s^{+}=y_{0} \\
s^{-} \geq 0, s^{+} \geq 0
\end{array}\right.
$$

If the optimal solutions of the model are $\lambda^{*}, s^{-*}$, $s^{+*}$ and $\theta^{*}$, then there are the following conclusions.

(1) If $\theta^{*}<1$, then $\mathrm{DMU}_{0}$ is not DEA effective. This scheme neither meets the requirements of the best technical efficiency nor the constant return of scale.

(2) If $\theta^{*}=1$, and at least one of $s^{-*}$ and $s^{+*}$ is not 0 , then $\mathrm{DMU}_{0}$ is weak DEA effective, that is, it is not both technically effective and scale effective.

(3) If $\theta^{*}=1$ and $s^{-*}=s^{+*}=0$, then $\mathrm{DMU}_{0}$ is DEA effective, that is, both technical efficiency and scale efficiency are satisfied.

\subsubsection{Technical effectiveness evaluation model (BCC)}

The BCC model is used to evaluate the relative technical effectiveness. Similarly, the calculation model can be obtained as follows. 
Table 1

MEMLS effectiveness evaluation indicators table

\begin{tabular}{lll}
\hline Target layer & First-level indicator layer & Second-level indicator layer \\
\hline Effectiveness evaluation of & Launch capability $U_{1}$ & Thrust control accuracy $U_{11}$ \\
MEMLS & Robustness during launch $U_{12}$ \\
& Maximum thrust $U_{13}$ \\
& Acceleration time $U_{14}$ \\
& Initial ejection velocity $U_{15}$ \\
& Infrared radiation intensity $U_{21}$ \\
& Electromagnetic anti-interference ability $U_{22}$ \\
& Electromagnetic compatibility of own system $U_{23}$ \\
& Initial anti-interception rate $U_{24}$ \\
& Energy utilization rate $U_{31}$ \\
& Ablation degree of the launcher $U_{32}$ \\
& Environmental pollution degree $U_{33}$ \\
& Spare parts replacement rate $U_{34}$ \\
& Ejection power unit weight $U_{41}$ \\
& Launcher weight $U_{42}$ \\
& Space-ratio performance $U_{43}$ \\
& Continuous combat capability $U_{44}$ \\
& Universality of launch system $U_{45}$ \\
\hline
\end{tabular}

$$
\left\{\begin{array}{c}
\max \left[\theta-\varepsilon\left(\hat{e}^{\mathrm{T}} s^{-}+\hat{e}^{\mathrm{T}} s^{+}\right)\right]=V \\
\text { s.t. } \sum_{k=1}^{s} \lambda_{k} x_{k}+s^{-}=\theta x_{0} \\
\sum_{k=1}^{s} \lambda_{k}=1 \\
\lambda_{k} \geq 0, s^{-} \geq 0, s^{+} \geq 0
\end{array}\right.
$$

If the optimal solutions of the model are $\lambda^{*}, s^{-*}$, $s^{+*}$ and $\theta^{*}$, when $\theta^{0}=1$ and $s^{-0}=s^{+0}=0, \mathrm{DMU}_{0}$ is technically effective, otherwise it is not technically effective.

\subsubsection{Scale effectiveness evaluation model}

Scale validity refers to verifying whether the DMU is at the optimal scale level, and it can be judged whether it is in a state of increasing, constant or decreasing scale. The scale efficiency of DMU is represented by $Q=\frac{\theta}{V}$. Calculate $K=\sum_{k=1}^{s} \lambda_{k}$. When $K=1$, the scale efficiency is unchanged; when $K<1$, the scale efficiency increases; when $K>1$, the scale efficiency decreases.

\section{Evaluation of MEMLS based on effectiveness}

\subsection{Effectiveness calculation based on GRA-PCA-LSSVM}

From the author's previous research [3], the effectiveness evaluation indicators of MEMLS can be obtained as shown in Table 1. From the related research of the subject, 64 sets of sample data for traditional launch methods can be obtained as shown in Table 2. Each set of data includes 18 evaluation indicators and the effectiveness value.

Due to the large number of indicators and the complexity of the evaluation method in literature [3], it needs to be simplified. First, perform a statistical description of the sample data as shown in Table 3, and make an indicator correlation strength diagram as shown in Fig. 2. The horizontal and vertical coordinates of Fig. 2 are indicator numbers, the color indicates the intensity of correlation, and the right side is the intensity and color contrast scale. It can be seen from Fig. 2 that there is a certain correlation between the indicators, so it can be considered to select and reduce the dimensionality of the indicators through GRA-PCA.

According to formulas (1)-(2), the correlation between each indicator and effectiveness is calculated, as shown in Table 4. The main indicators are selected with the degree of correlation $>0.7$, that is, $U_{11}, U_{12}, U_{13}, U_{15}, U_{22}, U_{24}, U_{31}$ and $U_{41}$.

After GRA, in order to further reduce the number of indicators and simplify the model, PCA was performed on the selected 8 indicators. According to Section 2.1.2, the correlation coefficient matrix is calculated as shown in Table 5, and the variance contribution rate of each principal component is calculated as shown in Table 6.

Here, the cumulative contribution rate $>90 \%$ is taken as the target, so 6 principal components need to be extracted, and the corresponding coefficients of 
Table 2

Effectiveness and indicators data of missile launch system

\begin{tabular}{|c|c|c|c|c|c|c|c|c|c|c|c|c|c|c|c|c|c|c|c|}
\hline \multirow[t]{2}{*}{ No. } & \multicolumn{19}{|c|}{ Indicators } \\
\hline & $\overline{U_{11}}$ & $U_{12}$ & $U_{13}$ & $U_{14}$ & $U_{15}$ & $U_{21}$ & $U_{22}$ & $U_{23}$ & $U_{24}$ & $U_{31}$ & $U_{32}$ & $U_{33}$ & $U_{34}$ & $U_{41}$ & $U_{42}$ & $U_{43}$ & $U_{44}$ & $U_{45}$ & $E$ \\
\hline 1 & 0.9 & 0.6 & 250 & 15 & 35 & 25 & 0.9 & 0.5 & 0.7 & 0.8 & 0.9 & 0.4 & 0.6 & 350 & 6 & 0.4 & 0.6 & 0.2 & 0.6371 \\
\hline 2 & 0.7 & 0.6 & 250 & 10 & 35 & 25 & 0.9 & 0.5 & 0.6 & 0.7 & 0.9 & 0.4 & 0.3 & 350 & 3 & 0.6 & 0.4 & 0.2 & 0.6239 \\
\hline 3 & 0.5 & 0.6 & 250 & 10 & 35 & 25 & 0.9 & 0.5 & 0.6 & 0.5 & 0.9 & 0.4 & 0.3 & 350 & 3 & 0.3 & 0.4 & 0.2 & 0.5851 \\
\hline 4 & 0.5 & 0.6 & 250 & 20 & 35 & 25 & 0.9 & 0.7 & 0.7 & 0.8 & 0.9 & 0.8 & 0.7 & 350 & 3 & 0.3 & 0.5 & 0.2 & 0.6669 \\
\hline 5 & 0.5 & 0.6 & 250 & 10 & 40 & 25 & 0.9 & 0.7 & 0.8 & 0.5 & 0.9 & 0.4 & 0.3 & 350 & 6 & 0.3 & 0.7 & 0.2 & 0.5997 \\
\hline 6 & 0.7 & 0.9 & 250 & 20 & 35 & 25 & 0.9 & 0.4 & 0.7 & 0.9 & 0.9 & 0.4 & 0.6 & 350 & 6 & 0.3 & 0.5 & 0.2 & 0.6152 \\
\hline 7 & 0.9 & 0.5 & 250 & 20 & 35 & 25 & 0.9 & 0.4 & 0.8 & 0.9 & 0.7 & 0.8 & 0.6 & 350 & 3 & 0.3 & 0.5 & 0.2 & 0.6534 \\
\hline 8 & 0.9 & 0.5 & 250 & 10 & 35 & 25 & 0.9 & 0.4 & 0.6 & 0.9 & 0.4 & 0.4 & 0.3 & 350 & 3 & 0.5 & 0.7 & 0.2 & 0.6059 \\
\hline 9 & 0.5 & 0.9 & 250 & 20 & 37 & 25 & 0.9 & 0.4 & 0.6 & 0.9 & 0.8 & 0.4 & 0.3 & 350 & 7 & 0.4 & 0.5 & 0.5 & 0.5896 \\
\hline 10 & 0.9 & 0.5 & 250 & 10 & 35 & 25 & 0.9 & 0.4 & 0.6 & 0.9 & 0.7 & 0.4 & 0.6 & 350 & 7 & 0.5 & 0.5 & 0.5 & 0.6361 \\
\hline 11 & 0.5 & 0.5 & 250 & 15 & 40 & 25 & 0.6 & 0.4 & 0.6 & 0.9 & 0.4 & 0.6 & 0.6 & 350 & 7 & 0.6 & 0.5 & 0.2 & 0.5514 \\
\hline 12 & 0.9 & 0.5 & 250 & 10 & 37 & 25 & 0.5 & 0.4 & 0.8 & 0.9 & 0.4 & 0.4 & 0.7 & 400 & 7 & 0.4 & 0.5 & 0.5 & 0.6015 \\
\hline 13 & 0.9 & 0.5 & 250 & 20 & 35 & 25 & 0.6 & 0.4 & 0.7 & 0.9 & 0.6 & 0.6 & 0.3 & 300 & 7 & 0.6 & 0.5 & 0.5 & 0.5741 \\
\hline 14 & 0.9 & 0.7 & 250 & 10 & 35 & 25 & 0.6 & 0.7 & 0.6 & 0.9 & 0.6 & 0.8 & 0.3 & 500 & 7 & 0.4 & 0.5 & 0.2 & 0.6240 \\
\hline 15 & 0.7 & 0.5 & 250 & 20 & 37 & 25 & 0.6 & 0.5 & 0.6 & 0.9 & 0.6 & 0.8 & 0.3 & 300 & 7 & 0.4 & 0.7 & 0.7 & 0.6026 \\
\hline$\ldots$ & $\ldots$ & $\ldots$ & $\ldots$ & $\cdots$ & $\ldots$ & $\cdots$ & $\cdots$ & $\cdots$ & $\ldots$ & $\ldots$ & $\ldots$ & $\ldots$ & $\cdots$ & $\ldots$ & $\cdots$ & $\ldots$ & $\ldots$ & $\ldots$ & \\
\hline 61 & 0.6 & 0.9 & 150 & 10 & 39 & 10 & 0.5 & 0.9 & 0.6 & 0.7 & 0.4 & 0.9 & 0.3 & 500 & 9 & 0.8 & 0.5 & 0.2 & 0.6366 \\
\hline 62 & 0.6 & 0.9 & 150 & 10 & 39 & 16 & 0.5 & 0.9 & 0.7 & 0.5 & 0.4 & 0.9 & 0.7 & 300 & 9 & 0.8 & 0.5 & 0.7 & 0.6847 \\
\hline 63 & 0.6 & 0.5 & 150 & 10 & 39 & 10 & 0.7 & 0.9 & 0.7 & 0.7 & 0.8 & 0.9 & 0.3 & 480 & 9 & 0.8 & 0.5 & 0.2 & 0.6637 \\
\hline 64 & 0.6 & 0.7 & 150 & 20 & 39 & 16 & 0.6 & 0.9 & 0.6 & 0.5 & 0.4 & 0.9 & 0.6 & 480 & 9 & 0.8 & 0.5 & 0.2 & 0.5855 \\
\hline
\end{tabular}

Table 3

Mathematical statistics of the original data of MEMLS

\begin{tabular}{lcccc}
\hline $\begin{array}{l}\text { Evaluation } \\
\text { Indicator }\end{array}$ & Minimum & Maximum & Average & $\begin{array}{c}\text { Standard } \\
\text { deviation }\end{array}$ \\
\hline$U_{11}$ & 0.50 & 0.90 & 0.6375 & 0.15379 \\
$U_{12}$ & 0.50 & 0.90 & 0.6547 & 0.16323 \\
$U_{13}$ & 150.00 & 250.00 & 195.4688 & 41.01605 \\
$U_{14}$ & 10.00 & 20.00 & 15.3906 & 4.07321 \\
$U_{15}$ & 35.00 & 40.00 & 37.0937 & 1.96573 \\
$U_{21}$ & 10.00 & 25.00 & 18.7812 & 6.62719 \\
$U_{22}$ & 0.40 & 0.90 & 0.6047 & 0.15779 \\
$U_{23}$ & 0.40 & 0.90 & 0.6156 & 0.16351 \\
$U_{24}$ & 0.60 & 0.90 & 0.7625 & 0.12536 \\
$U_{31}$ & 0.50 & 0.90 & 0.6844 & 0.16057 \\
$U_{32}$ & 0.40 & 0.90 & 0.5859 & 0.17716 \\
$U_{33}$ & 0.40 & 0.90 & 0.6141 & 0.18845 \\
$U_{34}$ & 0.30 & 0.80 & 0.4953 & 0.18724 \\
$U_{41}$ & 300.00 & 500.00 & 380.9375 & 72.93656 \\
$U_{42}$ & 3.00 & 9.00 & 5.8125 & 2.30166 \\
$U_{43}$ & 0.30 & 0.80 & 0.5297 & 0.15704 \\
$U_{44}$ & 0.40 & 0.90 & 0.5750 & 0.17817 \\
$U_{45}$ & 0.20 & 0.70 & 0.4000 & 0.21381 \\
\hline
\end{tabular}

6 principal components and 8 main indicators can be obtained as shown in Table 7.

According to Table 7 and formula (3), the calculation expression of the principal components can be obtained as follows.

In the formula: $U_{11}, U_{12}, U_{13}, U_{15}, U_{22}, U_{24}, U_{31}$ and $U_{41}$ represent normalized data values.

According to formula (15), Table 2 can be transformed into Table 8 .

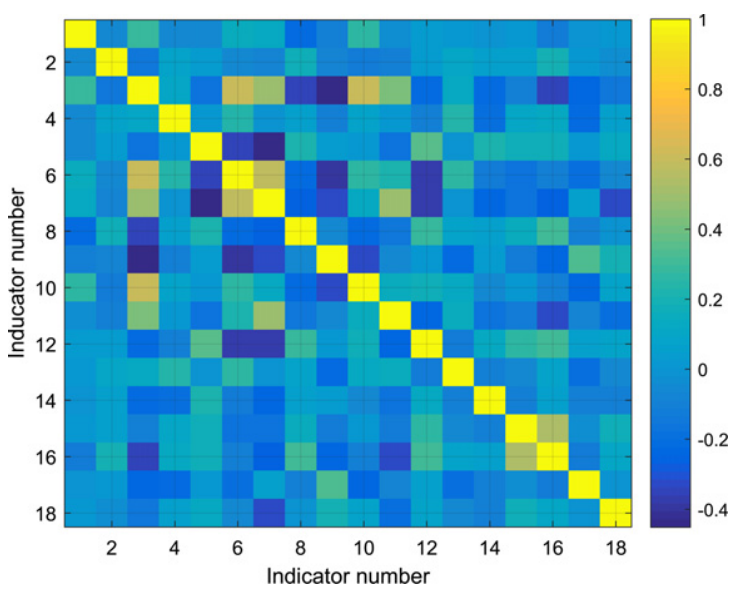

Fig. 2. Indicator correlation strength diagram.

$$
\begin{aligned}
Z_{1}= & 0.411 U_{11}-0.179 U_{12}+0.864 U_{13}-0.387 U_{15} \\
& +0.698 U_{22}-0.597 U_{24}+0.645 U_{31}-0.340 U_{41} \\
Z_{2}= & 0.285 U_{11}+0.024 U_{12}+0.182 U_{13}+0.704 U_{15} \\
& -0.449 U_{22}-0.234 U_{24}+0.521 U_{31}+0.471 U_{41} \\
Z_{3}= & -0.250 U_{11}+0.848 U_{12}-0.016 U_{13}-0.027 U_{15} \\
& +0.128 U_{22}-0.483 U_{24}-0.105 U_{31}+0.153 U_{41} \\
Z_{4}= & 0.772 U_{11}+0.314 U_{12}-0.102 U_{13}-0.225 U_{15} \\
& +0.029 U_{22}+0.264 U_{24}-0.099 U_{31}+0.206 U_{41} \\
Z_{5}= & 0.121 U_{11}+0.325 U_{12}+0.029 U_{13}+0.237 U_{15} \\
& -0.244 U_{22}+0.163 U_{24}+0.133 U_{31}-0.754 U_{41}
\end{aligned}
$$


Table 4

Correlation degree of each indicator and effectiveness

\begin{tabular}{lccccccccc}
\hline Indicator & $U_{11}$ & $U_{12}$ & $U_{13}$ & $U_{14}$ & $U_{15}$ & $U_{21}$ & $U_{22}$ & $U_{23}$ & $U_{24}$ \\
\hline correlation & 0.7165 & 0.7155 & 0.7206 & 0.6571 & 0.8688 & 0.5897 & 0.7112 & 0.6775 & 0.7616 \\
Indicator & $U_{31}$ & $U_{32}$ & $U_{33}$ & $U_{34}$ & $U_{41}$ & $U_{42}$ & $U_{43}$ & $U_{44}$ & $U_{45}$ \\
correlation & 0.7348 & 0.6675 & 0.6729 & 0.5925 & 0.7464 & 0.5944 & 0.6618 & 0.6647 & 0.4983 \\
\hline
\end{tabular}

Table 5

Correlation coefficient matrix of PCA

\begin{tabular}{cccccccccc}
\hline & & $U_{11}$ & $U_{12}$ & $U_{13}$ & $U_{15}$ & $U_{22}$ & $U_{24}$ & $U_{31}$ & $U_{41}$ \\
\hline Correlation & $U_{11}$ & 1.000 & -0.051 & 0.284 & -0.054 & 0.137 & -0.091 & 0.268 & -0.020 \\
coefficient & $U_{12}$ & -0.051 & 1.000 & -0.138 & 0.038 & -0.084 & -0.069 & -0.112 & 0.056 \\
& $U_{13}$ & 0.284 & -0.138 & 1.000 & -0.158 & 0.494 & -0.444 & 0.604 & -0.207 \\
& $U_{15}$ & -0.054 & 0.038 & -0.158 & 1.000 & -0.452 & 0.047 & 0.015 & 0.208 \\
& $U_{22}$ & 0.137 & -0.084 & 0.494 & -0.452 & 1.000 & -0.328 & 0.122 & -0.235 \\
& $U_{24}$ & -0.091 & -0.069 & -0.444 & 0.047 & -0.328 & 1.000 & -0.329 & 0.033 \\
& $U_{31}$ & 0.268 & -0.112 & 0.604 & 0.015 & 0.122 & -0.329 & 1.000 & -0.061 \\
& $U_{41}$ & -0.020 & 0.056 & -0.207 & 0.208 & -0.235 & 0.033 & -0.061 & 1.000 \\
\hline
\end{tabular}

Table 6

Variance contribution rate of PCA

\begin{tabular}{lccc}
\hline $\begin{array}{l}\text { Principal } \\
\text { component }\end{array}$ & Variance & $\begin{array}{c}\text { Contribution } \\
\text { rate / \% }\end{array}$ & $\begin{array}{c}\text { Cumulative contribution } \\
\text { rate / \% }\end{array}$ \\
\hline 1 & 2.472 & 30.899 & 30.899 \\
2 & 1.360 & 17.006 & 47.905 \\
3 & 1.067 & 13.336 & 61.241 \\
4 & 0.894 & 11.171 & 72.411 \\
5 & 0.850 & 10.622 & 83.034 \\
6 & 0.610 & 7.627 & 90.660 \\
7 & 0.480 & 5.999 & 96.659 \\
8 & 0.267 & 3.341 & 100.000 \\
\hline
\end{tabular}

$$
\begin{aligned}
Z_{6}= & -0.262 U_{11}+0.168 U_{12}+0.102 U_{13}-0.356 U_{15} \\
& -0.185 U_{22}+0.344 U_{24}+0.453 U_{31}+0.136 U_{41}
\end{aligned}
$$

The principal components are used as input, and the effectiveness is used as output, and regression fitting is performed. According to formulas (4)-(11), model training is performed and compared with PSOSVM and BP neural network algorithms. Select $85 \%$ of the samples as the training set and $15 \%$ of the samples as the test set. The fitting effect of the training set is shown in Fig. 3 and the fitting effect of the test set is shown in Fig. 4.

It can be intuitively obtained from Fig. 3 and Fig. 4 that the effect of the LSSVM model is better than that of PSOSVM and BP neural network. In order to get a specific comparison, calculate the mean square error (MSE) of the training set and the test set, as shown in Table 9.

It can be seen from Table 9 that the MSE of the training and test sets using the LSSVM model is the smallest, and the running time is the fastest, which can be further applied to the MEMLS design.

Each indicator value of the MEMLS design scheme is obtained from the research of the subject, and the effectiveness value is obtained through the pretrained LSSVM model, as shown in Table 10.

Table 7

Principal component coefficient

\begin{tabular}{lcccccc}
\hline Indicators & \multicolumn{7}{c}{ Principal component } \\
\cline { 2 - 7 } & 1 & 2 & 3 & 4 & 5 & 6 \\
\hline$U_{11}$ & 0.411 & 0.285 & -0.250 & 0.772 & 0.121 & -0.262 \\
$U_{12}$ & -0.179 & 0.024 & 0.848 & 0.314 & 0.325 & 0.168 \\
$U_{13}$ & 0.864 & 0.182 & -0.016 & -0.102 & 0.029 & 0.102 \\
$U_{15}$ & -0.387 & 0.704 & -0.027 & -0.255 & 0.237 & -0.356 \\
$U_{22}$ & 0.698 & -0.449 & 0.128 & 0.029 & -0.244 & -0.185 \\
$U_{24}$ & -0.597 & -0.234 & -0.483 & 0.264 & 0.163 & 0.344 \\
$U_{31}$ & 0.645 & 0.521 & -0.105 & -0.099 & 0.133 & 0.453 \\
$U_{41}$ & -0.340 & 0.471 & 0.153 & 0.206 & -0.754 & 0.136 \\
\hline
\end{tabular}


Table 8

Effectiveness and principal components data of MEMLS

\begin{tabular}{|c|c|c|c|c|c|c|c|}
\hline \multirow[t]{2}{*}{ No. } & \multicolumn{6}{|c|}{ Principal component } & \multirow[t]{2}{*}{ E } \\
\hline & $Z_{1}$ & $Z_{2}$ & $Z_{3}$ & $Z_{4}$ & $Z_{5}$ & $Z_{6}$ & \\
\hline 1 & 2.8840 & -0.4945 & -0.3521 & 1.1770 & 0.2571 & -0.3028 & 0.6371 \\
\hline 2 & 2.5919 & -0.9309 & 0.3985 & -0.0433 & 0.6588 & -0.5791 & 0.6239 \\
\hline 3 & 1.7415 & -1.8053 & 0.8395 & -0.9752 & 1.0095 & -0.8654 & 0.5851 \\
\hline 4 & 2.2044 & -1.1306 & 0.2770 & -0.9479 & 0.5988 & 0.5689 & 0.6669 \\
\hline 5 & 0.5092 & -0.5886 & 0.0280 & -1.2162 & 0.0737 & -1.3226 & 0.5997 \\
\hline 6 & 2.5900 & -0.4967 & 1.4089 & 0.6603 & -0.3102 & 0.8904 & 0.6152 \\
\hline 7 & 2.9061 & -0.3887 & -1.2914 & 1.1311 & 0.2423 & 0.2773 & 0.6534 \\
\hline 8 & 3.5122 & -0.0692 & -0.5458 & 0.6849 & 0.5242 & -0.4248 & 0.6059 \\
\hline 9 & 2.3028 & -0.0406 & 2.0698 & -0.9002 & -0.2601 & 0.5115 & 0.5896 \\
\hline 10 & 3.5122 & -0.0692 & -0.5458 & 0.6849 & 0.5242 & -0.4248 & 0.6361 \\
\hline$\ldots$ & $\ldots$ & $\ldots$ & $\ldots$ & $\ldots$ & $\ldots$ & $\ldots$ & $\ldots$ \\
\hline 61 & -1.1979 & 1.6010 & 2.0404 & 0.1202 & 0.6631 & -0.2537 & 0.6366 \\
\hline 62 & -1.4189 & -0.2224 & 1.3889 & -0.1226 & -1.5413 & -1.1040 & 0.6847 \\
\hline 63 & -0.5998 & 0.7927 & -0.2291 & -0.4926 & 1.4972 & -0.7784 & 0.6637 \\
\hline 64 & -1.2283 & 0.6651 & 1.1983 & -0.1970 & 1.2183 & -1.4377 & 0.5855 \\
\hline
\end{tabular}

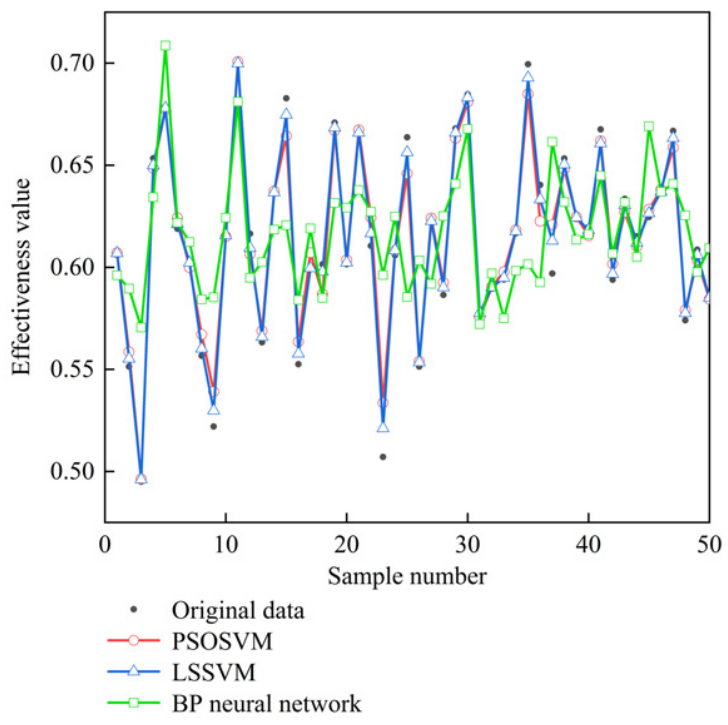

Fig. 3. Fitting diagram of the training set of effectiveness value.

\subsection{Analysis of investment efficiency based on DEA}

The whole life cycle cost and the cost of each stage of the MEMLS design scheme are obtained from the research of the subject. Obtain the effectiveness value from Table 10, and calculate the effectiveness -cost ratio, as shown in Table 11.

According to equations (12) - (14), the overall efficiency and pure technical efficiency of MEMLS are calculated, and its scale benefit and $\mathrm{K}$ value are calculated, as shown in Table 12.

According to Table 12, the following conclusions can be drawn.

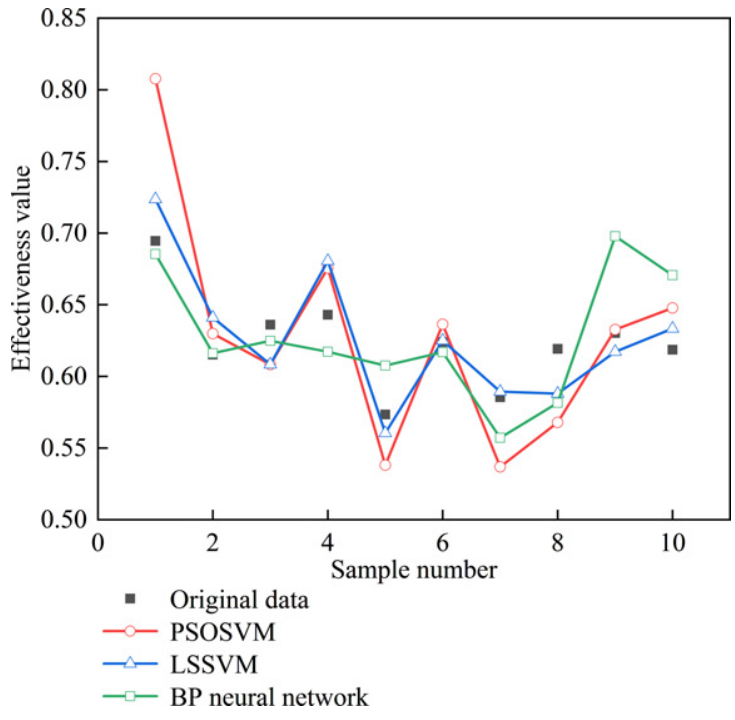

Fig. 4. Fitting diagram of the test set of effectiveness value.

Table 9

Performance comparison

\begin{tabular}{lccl}
\hline Performance & PSOSVM & LSSVM & $\begin{array}{l}\text { BP neural } \\
\text { netwok }\end{array}$ \\
\hline $\begin{array}{l}\text { Optimal } \\
\text { Parameters }\end{array}$ & Maxgen $=300$ & Gam $=400$ & $\begin{array}{l}\text { Hidden } \\
\text { layer }=10\end{array}$ \\
& Sizepop $=50$ & Sig2 $=5$ & $\begin{array}{l}\text { Learning } \\
\text { rate }=0.01 \\
\end{array}$ \\
& & & $\begin{array}{l}\text { MaxEpochs } \\
=5000\end{array}$ \\
& & & 0.0014 \\
Train-MSE & $7.8520 \mathrm{e}-05$ & $2.0358 \mathrm{e}-05$ & 0.0012 \\
Test-MSE & 0.0022 & $5.2525 \mathrm{e}-04$ & $0.535 \mathrm{~s}$ \\
Time & $66.009 \mathrm{~s}$ & $0.710 \mathrm{~s}$ \\
\hline
\end{tabular}


Table 10

Design indicators and effectiveness values of MEMLS

\begin{tabular}{lccccccccc}
\hline Scheme Number & $U_{11}$ & $U_{12}$ & $U_{13}$ & $U_{15}$ & $U_{22}$ & $U_{24}$ & $U_{31}$ & $U_{41}$ & $E$ \\
\hline 1 & 0.9 & 0.8 & 200 & 37 & 0.9 & 0.8 & 0.8 & 350 & 0.6779 \\
2 & 0.8 & 0.9 & 200 & 37 & 0.7 & 0.8 & 0.9 & 350 & 0.6082 \\
3 & 0.8 & 0.8 & 250 & 37 & 0.7 & 0.8 & 0.8 & 300 & 0.6416 \\
4 & 0.8 & 0.8 & 200 & 37 & 0.7 & 0.9 & 0.9 & 350 & 0.6665 \\
5 & 0.8 & 0.8 & 200 & 37 & 0.7 & 0.8 & 0.8 & 350 & 0.5564 \\
\hline
\end{tabular}

Table 11

Cost and effectiveness data of MEMLS

\begin{tabular}{|c|c|c|c|c|c|c|}
\hline \multirow{2}{*}{$\begin{array}{l}\text { Scheme } \\
\text { Number }\end{array}$} & \multicolumn{4}{|c|}{ Investment indicators (100 million yuan) } & \multicolumn{2}{|c|}{ Output indicators } \\
\hline & $\begin{array}{l}\text { Development } \\
\text { cost }\end{array}$ & $\begin{array}{l}\text { Production } \\
\text { cost }\end{array}$ & $\begin{array}{c}\text { Use and } \\
\text { guarantee cost }\end{array}$ & $\overline{L C C}$ & $E$ & $E / L C C$ \\
\hline 1 & 1.2 & 3.5 & 7.2 & 11.9 & 0.6779 & 0.0570 \\
\hline 2 & 1.2 & 3.6 & 6.5 & 11.3 & 0.6082 & 0.0538 \\
\hline 3 & 1.4 & 3.8 & 6.0 & 11.2 & 0.6416 & 0.0573 \\
\hline 4 & 2.2 & 3.8 & 6.9 & 12.9 & 0.6665 & 0.5167 \\
\hline 5 & 1.6 & 4.0 & 5.5 & 11.1 & 0.5564 & 0.0501 \\
\hline
\end{tabular}

Table 12

Calculation results using DEA model

\begin{tabular}{lcccc}
\hline $\begin{array}{l}\text { Scheme } \\
\text { Number }\end{array}$ & $\begin{array}{c}\text { overall } \\
\text { efficiency }\end{array}$ & $\begin{array}{c}\text { pure technical } \\
\text { efficiency }\end{array}$ & $\begin{array}{c}\text { scale } \\
\text { benefit }\end{array}$ & $K$ \\
\hline 1 & 1 & 1 & 1 & 1 \\
2 & 0.9599 & 1 & 0.9599 & 0.9117 \\
3 & 1 & 1 & 1 & 1 \\
4 & 1 & 1 & 1 & 1 \\
5 & 0.9462 & 1 & 0.9462 & 0.8672 \\
\hline
\end{tabular}

(1) From the perspective of overall efficiency, schemes 1, 3, and 4 are all effective, and schemes 2,5 have efficiency values $<1$, and there is room for adjustment.

(2) From the point of view of pure technical efficiency, the five programs are all at a relatively high level.

(3) From the perspective of returns to scale, schemes 1, 3, and 4 remain unchanged and are the optimal level of investment scale, while schemes 2, 5 are incremental and need to be adjusted.

Therefore, the cost of the MEMLS design scheme is adjusted, and the results are shown in Table 13.

\section{Conclusion}

This paper takes MEMLS as the research object. Based on the previous research, a fast calculation model based on GRA-PCA-LSSVM is established, and the rationality and superiority of the calculation model are verified. At the same time, based on the DEA model, the investment efficiency of the MEMLS scheme is analyzed. The conclusions of this paper are as follows:

(1) Through GRA-PCA, the main indicators of system evaluation can be effectively extracted and the dimensionality of the input vector can be reduced, which is reasonable and necessary.

(2) LSSVM can effectively construct the performance prediction model of MEMLS. Compared with other methods, this model has higher accuracy and shorter calculation time.

(3) Through the DEA model, the input and output of the MEMLS program can be adjusted, and the method is effective and feasible.

(4) In the existing 5 design schemes of MEMLS, it is necessary to adjust the life cycle cost of schemes 2 and 5 to achieve the best investment scale level.

Cost adjustment plan of MEMLS (100 million yuan)

\begin{tabular}{lcccc}
\hline $\begin{array}{l}\text { Scheme } \\
\text { Number }\end{array}$ & $\begin{array}{c}\text { Development } \\
\text { cost }\end{array}$ & $\begin{array}{c}\text { Production } \\
\text { cost }\end{array}$ & $\begin{array}{c}\text { Use and } \\
\text { guarantee cost }\end{array}$ & LCC \\
\hline 2 & 1.2000 & 3.4176 & 6.5000 & 11.1176 \\
5 & 1.3009 & 3.5106 & 5.5000 & 10.3116 \\
\hline
\end{tabular}


In the next step, the specific scheme design and deployment scale of MEMLS will be studied.

\section{References}

[1] Y. Lu, H. Jiang, T. Liao, C. Xu and C. Deng, Characteristic Analysis and Modeling of Network Traffic for the Electromagnetic Launch System, Mathematical Problems in Engineering 1 (2019), 1-7.

[2] C. Deng, C. Ye, J. Yang, D. Yu and S. Sun, et al., A Novel Permanent Magnet Linear Motor for the Application of Electromagnetic Launch System, IEEE Transactions on Applied Superconductivity 99 (2020), 1-1.

[3] Q. Li, G. Chen, L. Xu, Z. Lin and L. Zhou, An Improved Model for Effectiveness Evaluation of Missile Electromagnetic Launch System, IEEE Access 8 (2020), $156615-156633$.

[4] X. Wang, J. Zhao, Q. Li, N. Fang and S. Li, A hybrid model for prediction in asphalt pavement performance based on support vector machine and grey relation analysis, Journal of Advanced Transportation 2 (2020), 1-14.

[5] Y. Yu, M. J. Peng, H. Wang, Z.G. Ma and W. Li, Improved PCA model for multiple fault detection, isolation and reconstruction of sensors in nuclear power plant, Annals of Nuclear Energy 148 (2020), 107662.

[6] H. Chung and K.S. Shin, Genetic algorithm-optimized multi-channel convolutional neural network for stock market prediction, Neural Computing and Applications 1 (2020).

[7] T. Stoichev, J.P. Coelho, A.D. Diego, M.G.L. Valenzuela and D. Amouroux, Multiple regression analysis to assess the contamination with metals and metalloids in surface sediments (aveiro lagoon, portugal), Marine Pollution Bulletin 159 (2020).

[8] W. Liu, G. Guo, F. Chen and Y. Chen, Meteorological pattern analysis assisted daily PM2.5 grades prediction using SVM optimized by PSO algorithm, Atmospheric Pollution Research, 2019.

[9] L.L. Yeung and P.F. Azevedo, Measuring efficiency of brazilian courts with data envelopment analysis (DEA), Ima Journal of Management Mathematics 4 (2018), 343-356.

[10] W. Yang and L. Li, Efficiency evaluation of industrial waste gas control in china: a study based on data envelopment analysis (DEA) model, Journal of Cleaner Production 179 (2018), 1-11.

[11] J. Zhen, The diferential grey model(GM) and its implement in long period forecasting of grain, Discovery of Nature $\mathbf{3}$ (1984).

[12] D. Loganathan, S.S. Kumar and R. Ramadoss, Grey relational analysis-based optimisation of input parameters of incremental forming process applied to the aa6061 alloy, Transactions of FAMENA 1 (2020), 93-104.

[13] B. Deng and Y. Shi, Modeling and optimizing the composite prepreg tape winding process based on grey relational analysis coupled with BP neural network and bat algorithm, Nanoscale Research Letters 14 (2019).

[14] X. Hui, Y. Sun, F. Yin and W. Dun, Trend prediction of agricultural machinery power in china coastal areas based on grey relational analysis, Journal of Coastal Research 103 (2020), 299.

[15] Z. Zuka, J. Kopcińska, E. Dacewicz, B. Skowera, J. Wojkowski and A. Ziernicka-Wojtaszek, Application of the principal component analysis (PCA) method to assess the impact of meteorological elements on concentrations of particulate matter (PM 10): a case study of the mountain valley (the scz basin, poland), Sustainability 11 (2019).

[16] D. Ruiz, B. Bacca and E. Caicedo, Hyperspectral images classification based on inception network and kernel PCA, IEEE Latin America Transactions 12 (2019), 1995-2004.

[17] P.J. Papandrea, E.P. Frigieri, P.R. Maia, L.G. Oliveira and A.P. Paiva, Surface roughness diagnosis in hard turning using acoustic signals and support vector machine: a PCAbased approach, Applied Acoustics 107102 (2019).

[18] J.A.K. Suykens and J. Vandewalle, Least Squares Support Vector Machine Classifiers, Kluwer Academic Publishers 3 (1999), 293-300.

[19] X. Zhu, S.Q. Ma, Q. Xu and W.D. Liu, A WD-GA-LSSVM model for rainfall-triggered landslide displacement prediction, Journal of Mountainence 1 (2018), 156-166.

[20] J. Duan, X. Qiu, W. Ma, X. Tian and D. Shang, Electricity consumption forecasting scheme via improved LSSVM with maximum correntropy criterion, Entropy 2 (2018).

[21] R. Razavi, A. Sabaghmoghadam, A. Bemani, A. Baghban and E. Salwana, Application of anfis and lssvm strategies for estimating thermal conductivity enhancement of metal and metal oxide based nanofluids, Engineering Applications of Computational Fluid Mechanics 1 (2019), 560-578.

[22] F. Li, Q. Zhu and L. Liang, A new data envelopment analysis based approach for fixed cost allocation, Annals of Operations Research 1 (2019), 1-26.

[23] B.D. Rouyendegh, A. Yildizbasi and I. Yilmaz, Evaluation of retail ndustry performance ability through ntegrated ntuitionistic fuzzy topsis and data envelopment analysis approach, Soft Computing 2 (2020).

[24] H. Fazlollahtabar, Comparative simulation study for configuring turning point in multiple robot path planning: robust data envelopment analysis, Robotica (2019), 1-15.

[25] X. Chan, M. Yiyuan, H. Xiaobin, X. Renqiao and S.O. Management, A study on operational efficiency of hi-tech startups in china based on DEA methods, Journal of Managementence 2014. 